\title{
A Versatile Gripper for Cloth Manipulation
}

\author{
Sònia Donaire, Júlia Borràs, Guillem Alenyà, Carme Torras
}

\begin{abstract}
Cloth manipulation has been mostly advancing in perception and modeling methods for cloth state estimation and grasping point detection. In comparison, less attention has been put on end-effector design. Indeed, most implementations use a parallel gripper that can only perform pinch grasps. Instead, a more versatile set of possible grasp types could ease many tasks by providing more support to certain parts of the object, as well as make feasible tasks that become very complex when executed with only pinch grasps. We present a versatile gripper design which, besides the common open-close thumb+finger(s) feature, it has a couple of reconfiguration degrees of freedom that offer, first, a wide base plane to provide support and, second, variable friction surface on the thumb tip. Our gripper can execute a versatile set of grasps that ease some complex tasks such as pick and place folded clothes or fold in the air. In addition, the variable friction mechanism enables a more robust pinch-and-slide manipulation to trace cloth edges. Our evaluation shows the gripper potential to execute a wide variety of cloth manipulation tasks.
\end{abstract}

\section{INTRODUCTION}

Manipulation of cloth is the last frontier of general robotic manipulation. Despite clothes and fabrics are very common in our environment, robotic research on cloth manipulation is still in its early stages due to the great challenges it involves. Perception methods have to deal with self-occlusions and difficulties in understanding a bundled piece of cloth. The lack of models to predict cloth behaviour under touch make it very difficult to close the loop during manipulation and to monitor task execution. In this context, it is understandable that most of the efforts have been put on developing solutions for cloth state estimation and grasping point detection. A good review of the state-of-the-art of these methods can be found in [1]. One of the conclusions of the review is that there has been many advances in perception methods but, in comparison, manipulation skills remain underdeveloped. and that there is a need of increasing the dexterity of endeffectors by improving the current hardware.

Indeed, there has been very little attention on the study of different grasp types to manipulate cloth. A simple inspection of humans performing cloth manipulation shows how many different grasps we realize when we manipulate cloth [2]. However, most of the popular cloth manipulation works such as [3-7] use simple parallel grippers that can only perform a pinch grasp. Therefore, it seems just obvious to think the

This work receives funding from the European Union Horizon 2020 Programme under grant agreement no. 741930 (CLOTHILDE), Spanish State Research Agency through the María de Maeztu Seal of Excellence to IRI (MDM-2016-0656) and the "Ramon y Cajal" Fellowship RYC-201722703.

The authors are with Institut de Robòtica i Informàtica Industrial, CSIC-UPC, Llorens i Artigas 4-6, 08028 Barcelona, Spain. \{jborras, torras, galenya\}eiri.upc.edu



Fig. 1. The cloth gripper has a thumb finger $f_{1}$ rotating around the $u_{1}$ axis, a fixed finger $f_{2}$ and a third abducting finger $f_{3}$ rotating around the $u_{3}$ axis. The third degree of freedom is powered by a small motor that pushes out a high-friction surface on the thumb tip, or hides it, along the direction $v_{1}$. Finger $f_{3}$ can abduct up to an angle $a=40^{\circ}$. Bottom fingers have additional passive compliant rotation around $u_{4}$ and $u_{5}$ only in the shown direction.

complexity of manipulating a piece of cloth could be eased by having more support geometries to assist the motion. With this idea in mind, our previous work [8] proposes a new framework to define different grasp types for clothes and reviews cloth manipulation tasks focusing on grasping.

In this work, we design and build a gripper prototype for cloth manipulation based on the requirements of three manipulation tasks: pick and place folded clothes, fold a Tshirt in the air, and unfold in the air by tracing an edge by performing a pinch-and-slide manipulation. These three tasks have been chosen because they are difficult to execute with just a pinch grasp. We first review the literature on how these tasks have been approached so far, and then we extract the grasping and functionality requirements using our grasp framework based on the geometries of the two virtual fingers that perform the grasp. The requirements lead to a mechanical design solution that has been built with rapid prototyping tools (see Fig. 1) and tested by performing the three set of tests holding the gripper with our hands. Future work include using the gripper with a robot arm and 
improving the design with extra functionalities to deal also with rigid objects.

\section{RELATED WORK}

There is an extensive bibliography on industrial grasping devices for textiles, a good review can be found in [9]. However, they are mostly based on vacuum, needles, air-jets and adhesive technologies that, in general, can be destructive for the garment and are usually designed for industrial environments. Gripper designs based on contact and friction can be more generic, but those specially designed for handling clothes are much less common due to the unique challenges for grasping and manipulation of textiles. Some examples include [10-16]. However, most of these grippers solve only partial problems or particular specific tasks on cloth manipulation. In addition, they can rarely be used to grasp other objects.

For instance, [10] presents the Clopema EU project gripper, used to pick up garments from a pile and folding them. Their study concludes that small fingertips are required to pinch clothes from the pile, and flat and thin fingertips are required to clamp a flat cloth on a table from an edge. In addition, the gripper is equipped with specially-designed tactile sensor at the tip. The gripper is shown to be very useful to execute cloth manipulation tasks in [6], but the gripper is not very versatile. It can only perform a pinch grasp, and a linear flattening over the table. But it would have difficulties to grasp other types of rigid objects or even piles of folded clothes.

Some more general grippers for cloth manipulation appear in $[11,12]$. Ono et al. in [12] present a humanoid hand with 2 fingers, a thumb and a palm. It is one of the few designs that can realize different types of grasps on cloth, at the expense of resulting in a very complex design close to a humanoid hand. The design in [11] is an underactuated hand with a simpler and smart design, optimized for grasping clothes that are laying flat on a table. However, it cannot realize other important tasks like flattening a cloth on a table.

Many gripper designs focus on the complex pinch-andslide edge tracing manipulation. This manipulation consists in grasping an edge of a cloth and sliding over it until the corner is reached. It has been used only on very small clothes in [7] with an off-the-shelf gripper due to its complexity, but it has the potential to simplify the unfold in the air task. Therefore, many gripper designs try to ease this task. For instance, in [16] the authors present a gripper that consists of two small parallel grippers joined by a prismatic actuator that moves the two grippers apart, allowing to execute the pinch-and-slide. A similar design appears in [17]. The main limitation of this type of grippers is that they can only "unfold" clothes as long as the gripper or shorter and that they are very bulky for a domestic environment. In [15] a parallel gripper is specially designed to execute the task of tracing an edge using rolling fingertips. A later design [14] realizes edge tracing with an inchworm strategy, that is, a couple of grippers that are always grasping the fabric but performing a grasp gaiting that allows to translate along the edge. These are interesting designs, but they do not explore other possible ways of grasping the clothes for other tasks.

In the recent years there has been an increasing interest in designing mechanisms that can change their friction properties, mainly utilizing properties of soft materials [1821]. It has been shown that variable friction can increase the ability of simple rigid 2-finger grippers to perform in-hand manipulations $[18,21]$. Some designs try to replicate the human fingertips properties where more pressure brings more friction, using a smart shape fingertip with soft materials [20] or a simpler but effective passive mechanism [18]. The mechanism in [19] can tune the friction through an inflatable membrane that is pushed out through fingertip holes. The main challenge we face to utilize these mechanisms for our case is that fabric naturally has very high friction with certain silicone materials, even when the surface contact is small like in [20] and the softness of the material makes it hard to prevent contact with the high friction material even when it is turned off. That would be the case in designs like [18, 20, 21]. It could work on a mechanism like [19] but it requires pneumatic actuation. Therefore, we propose a variation of the Spiers et al. design [18]. In our design the high friction surface is the one that hides inside the finger while the rest of the finger surface is low friction, just the opposite of Spiers et al. design. In the evaluation, we show that a small highfriction surface is enough to strongly hold a fabric, and when is hidden the fabric is not in contact with it and the fabric can slide inside the gripper.

We present a gripper design able to perform a list of tasks that require versatile grasp types. Our main contribution is to design a general cloth manipulation gripper that is able to go beyond the pinch grasp and that can satisfy several very different requirements. As we concentrate on clothing manipulation, the presented design is not suitable for rigid objects at the current state. However, we have considered to add an additional degree of freedom in the thumb for a future design, to allow rigid object grasping and manipulation in a similar way as the design in [22].

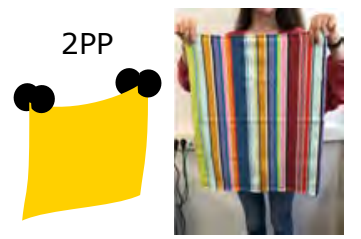

(a)

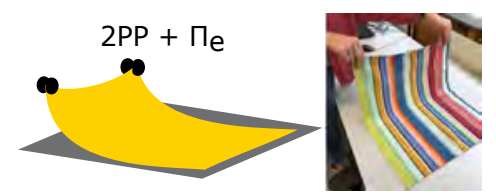

(b)

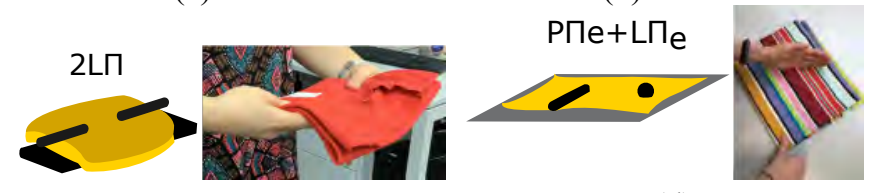

(c)

(d)

Fig. 2. Point contacts are noted as P, linear contacts as L and planar contacts as $\Pi$. (a) Double pinch grasp. Each pinch is formed with two point geometries. (b) A double pinch with the additional extrinsic contact of the table. (c)A double grasp obtained with a linear contact and a planar surface, called a (LI) grasp (d) A more complex combination of grasps achieved with a grasping agent (the hand) against the table. 


\section{GRIPPER REQUIREMENTS DEFINED FROM TASK SPECIFICATIONS}

From our previous works [8], we could conclude that cloth has been manipulated through mostly pinch grasps. This has worked well for tasks such as picking up crumpled clothes, manipulating a cloth alternating pinch grasps between each hand, putting a cloth flat on a table and folding it. Although these represent a very important set of tasks in cloth manipulation, there are many others that would be difficult to achieve with only pinch grasps. For instance, flattening a cloth on a table already requires alternative geometries that are usually supplied with the surfaces on the sides of grippers $[3,6]$.

In our previous work [8] we introduced a cloth grasp framework where each grasp was defined by the geometries of the two contact patches that perform the couple of opposing forces, that is, the geometries of each virtual finger [23, 24]. The framework considered virtual fingers geometries that could be either intrinsic (part of the gripper) or extrinsic (part of the environment). A brief idea of grasp definitions is shown in Fig. 2. In this work we will use the framework to identify what geometries our gripper needs to realize.

We will focus our design on three tasks that require a wider variety of grasps, in addition to the pinch grasp. These are: pick and place folded clothes, fold in the air and trace an edge.

\section{A. Task 1: Pick and place folded clothes}

Once a cloth is already folded, manipulating it requires to maintain the folded state, usually by a platform grasp under it, combined with an additional side grasp. This task has been done with pinch grasps in the past. In [3] we could see PR2 picking up folding towels with just two pinch grasps. However, in the video you can clearly see that, despite manipulating quite thick and rigid garments, after the folded towels were placed on the table, they needed an additional flattening. This was done as an open-loop procedure. A more informed approach was recently published [25], where a single parallel gripper was used. Their approach has the limitations that they need to localize the thickest folded hem and they do not solve the problem of placing back the cloth without unfolding it. A more obvious solution is to slide under the cloth with a flat, wide and thin surface, that we will call a plane $(\Pi)$. Once the plane is under the garment the cloth can be already transported without being unfold. An additional upper finger can be used to ensure the cloth doesn't fall, with either a linear or a point contact. We call this grasp a Line-Plane (LI) ( Fig. 2-(c) ). A single nonprehensile $\Pi$ grasp can also transport a pile of folded clothes.

\section{B. Task 2: Fold a T-shirt in the air}

Compared to fold a T-Shirt on a table with a double pinch grasp as in Fig. 2-(b), folding a T-shirt in the air can be done much faster. This is because it consists of a single, although more complex, manipulation. Due to its velocity potential, this task is of great interest for several applications,

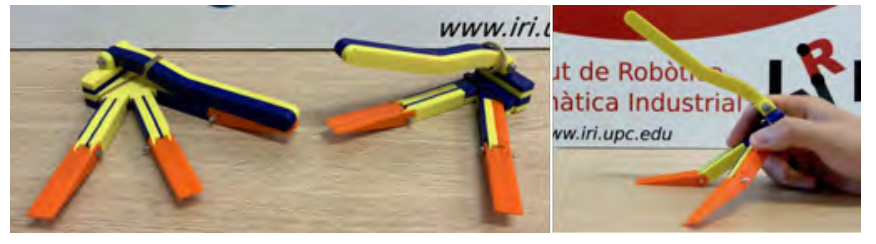

Fig. 3. Simple prototypes to evaluate the grasp geometries.

for instance, inverse logistics automated solutions for online cloth retailers.

Existing partial solutions in literature have used only pinch grasps. In [26] they used an open-loop procedure to fold a Tshirt where clearly the sleeves where not correctly folded. A more informed method was used in [27] with reinforcement learning, assuming a piece already grasped and without performing the release. Attempting this operation with only point contacts (a pinch grasp) is very challenging and requires a lot of dynamics. Using a linear or planar contact along the shoulders of the T-shirt allows to transmit the torque much more efficiently to perform the correct fold in the air, hold it under gravity and then add the contact with the table to finish the operation. Therefore, we need to realize a linear or a planar geometry along the shoulder.

\section{Task 3: Trace an edge}

Once a rectangular cloth is grasped by one of its corners, we could grasp one of the adjacent edges with the other hand and slide it until the other corner is reached. This is a complex operation that has been only approached with robots in [7], with very small clothes. However, it becomes clear that to perform the same manipulation in a bigger cloth, such as a big towel or a tablecloth, the two grasps involved require very different properties. To grasp the corner, the pinch grasp has to be strong and support the possible pulls due to the sliding. That means it requires either a high friction at the fingertips or a lot of motor force. On the contrary, the hand that slides along the edge needs a low friction, until it reaches the corner and a strong grip is required again. This is just an example task where different frictions are needed. Another task is tucking a cloth under a mattress when you fit an elastic cover, as pointed out in [13].

\section{General cloth manipulation}

In addition to the specific tasks, we want our gripper to be able to perform generic cloth manipulation.Therefore, we require the widely used pinch grasp, but also to be able to slide under a flat cloth, that is, to have thin fingertips and certain compliance to account for positional errors of the gripper to touch the table. Another very common task is to flatten a cloth against a table. For that, one needs a planar or linear geometry that can be slid over the surface of the flat cloth. In our framework the table is the extrinsic virtual finger $\Pi_{e}$, and the flattening grasps are $\Pi_{e}$ or $\mathrm{L}_{e}$.

\section{GRIPPER REQUIREMENTS AND PROPOSED SOLUTIONS}

From the analysis of the tasks above, we can extract the following requirements: 
TABLE I

GRASP GEOMETRIES IMPLEMENTATION

\begin{tabular}{c|c|c|c|} 
Grasp type & Implementation \\
(Point-Point) \\
$\begin{array}{c}\text { LI } \\
\text { (Line-Plane) }\end{array}$
\end{tabular}

Notes: Following the notation from Fig. 2 P stands for point, L for line and $\Pi$ for plane. $\Pi_{e}$ stands for extrinsic plane

- Grasps:

- Prehensile: $\{\mathrm{P} \Pi, \mathrm{L} \Pi$, or $\Pi \Pi\}$ for the pick and place, $\{L L, P L\}$ for the folding in the air and PP for the edge tracing.

- Non-prehensile: П (also for the pick and place).

- With extrinsic virtual fingers: $\Pi_{e}$ or $\mathrm{L \Pi}_{e}$.

- Functionalities:

- Thin fingertips at the bottom virtual fingers, with the option of flexibility to account for positional errors.

- Thin edge of the planar virtual finger, to go under the folded garment.

- Variable friction for the PP grasp.

We worked with the idea of a simple 2-finger gripper prototype with a joint-less bottom finger realizing a planar surface, while the top finger realizing a linear contact. A plane at the bottom would easily realize the $\Pi_{e}$ grasp against a table. The edge of the bottom plane could be used to perform a $\mathrm{L}_{e}$ grasp and we formed the planar bottom with a set of abducted fingers.

Our preliminary set of experiments were meant to test the geometries and evaluate if an abduction/adduction degree

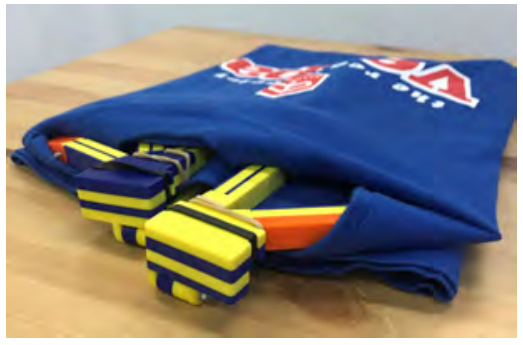

Fig. 4. Releasing the folded T-shirt without closing the abducted fingers was difficult.

of freedom for the bottom fingers was necessary. To do so, we built very simple prototypes that could be actuated with our hands with simple rubber bands (Fig. 3). One prototype realized the bottom plane with 3 fixed fingers, while the other with only 2 . In addition, the thin fingertips have one passive compliant joint to account for position errors when touching the table, as shown in Fig. 3 right, but they are rigid when touched from above.

All the tasks were carried out by manipulating the clothes with the simple prototypes. Results of the grasp realizations are shown in Table I. Although this is not enough to ensure a full prototype would work, it was useful to test if the geometries were enough, estimate correct dimensions and find additional requirements. We concluded that when folding in the air, the geometry of the gripper was giving good support for the manipulation, but after the T-shirt was folded, it was almost impossible to remove the grippers without reducing the space between the abducted fingers (see Fig. 4p. Therefore, actuation to open and close the abduction of the bottom fingers was necessary. Our second conclusion was that the use of the third finger was not adding any functionality to the gripper, but it was increasing a lot its complexity, especially if actuation was needed to adduct/abduct the fingers.

Finally, the last requirement was to have variable friction. To solve this issue, the last phalanx of the top finger has a mechanism inspired in [18], with a much smaller design, where the exterior surface is the 3D printed material that has a low friction, and there is a hidden part with a high friction surface that can be pushed out by some small motors. The dimensions of prototype with two fingers at the base were kept in terms of length and wideness of the opening, but the thickness of the fingers had to be increased to fit the mechanism of variable friction, resulting in the final prototype shown in Fig. 1.

\section{PROTOTYPE IMPLEMENTATION}

In this section, we describe the main characteristics of the mechanical design. However, full detailed instructions for building and assembling the gripper, together with the CAD models, can be found at the provided website ${ }^{1}$. Passive compliance at the bottom fingers is achieved with a compliant metal plate inside the fingers, and a geometric design

\footnotetext{
${ }^{1}$ http://www.iri.upc.edu/groups/perception/\#clothGripper Please, check website address spelling if the link doesn't work.
} 


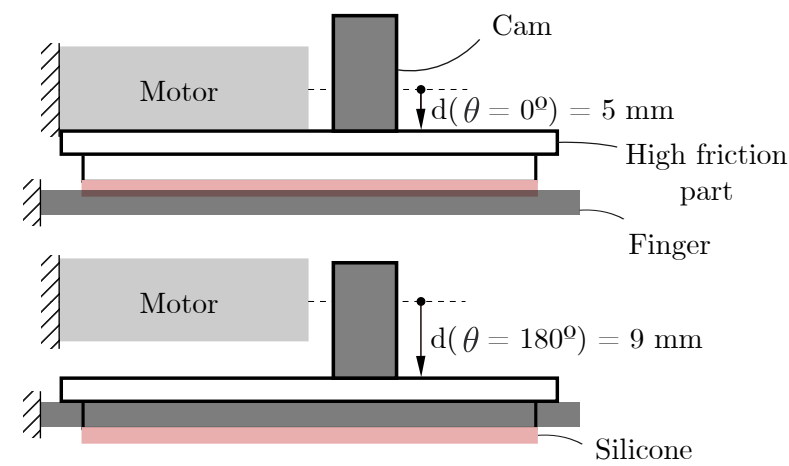

Fig. 5. Schematic drawing of the cam mechanism. In the top figure, the two friction bands (in red) are hidden, and in the bottom figure, the cam is rotated $180^{\circ}$, pushing down the high friction bands through the holes. At the top-right we show the profile of the cam.

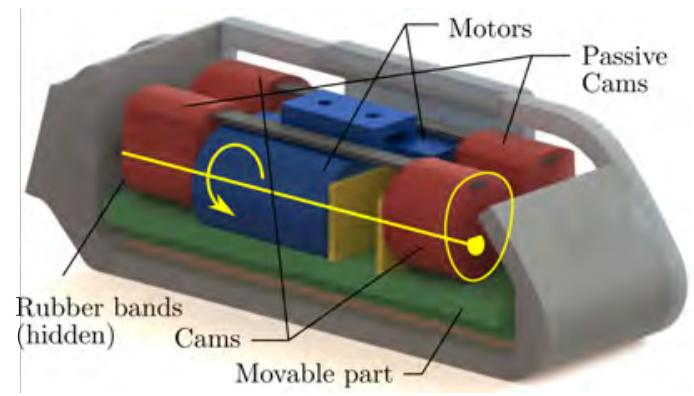

Fig. 6. CAD design of the cam mechanism mounted at the distal phalanx of the top finger. The red parts are the cams, in green is the movable part that has the high-friction bands attached. In blue, the part that contains the motors.

of the middle joint that prevents the degree of flexion from exceeding $30^{\circ}$ (like in Fig. 3 -right).

Most of the gripper complexity is in its top finger. It contains two phalanges, accounting for a future degree of freedom that has remained fixed in this prototype version. The finger geometry allows a more stable grip due to the larger contact surface provided by the distant phalanx, that is in full contact when the hand is closed. This distal phalanx contains the variable friction mechanism.

To implement the variable friction mechanism, the finger has a low friction surface in which two holes have been made. A cam mechanism actuates a movable part with two high-friction bands that come out through the holes when the cams are rotated (see Fig. 5). Two elastic bands ensure the movable part follows the cam maintaining always contact. In addition, a passive cam is added at the other side of the motor that moves always in accordance providing another contact point to the movable part. In order to facilitate that the surfaces with high friction go out through the holes without problems and always in parallel, two systems like the one in Fig. 5 have been placed in parallel, as shown in Fig. 6 . Therefore, in total we have two active cams actuated by two identical motors and two passive ones. The advantage of using this kind of mechanism is that, once the cams are in position, either at $180^{\circ}$ or at $0^{\circ}$, the motors do not need to counteract any gripping force. Therefore, they just

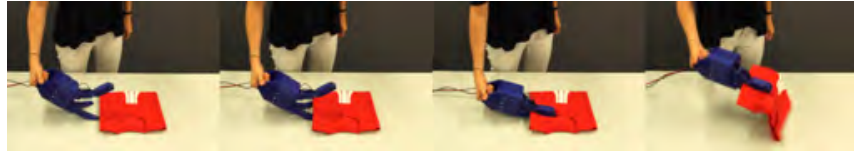

a) Pick and place folded clothes (single hand).

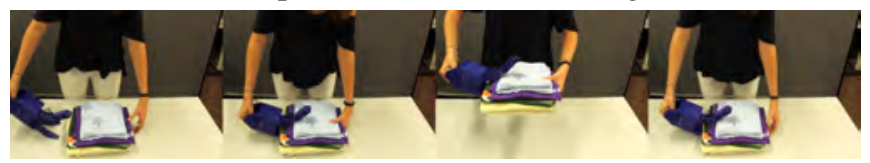

b) Pick and place a pile of clothes (bi-manual).

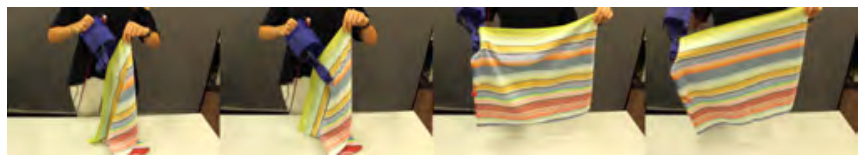

c)Trace an edge by pinching and sliding (friction hidden).

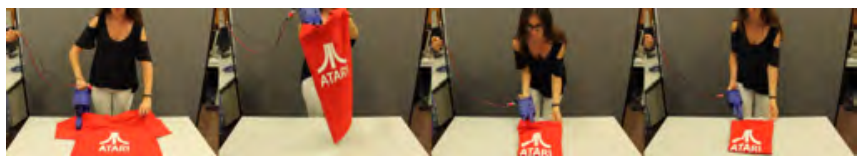

d) Fold a T-shirt in the air (abducted fingers and friction to fold, adducted and no friction to release).

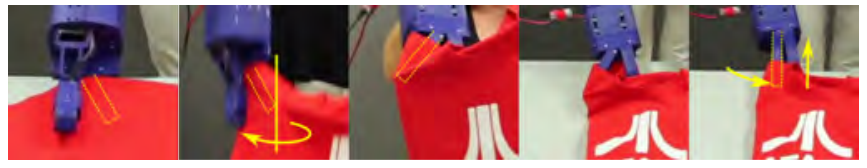

e) Detail of the hand manipulation during folding in the air.

Fig. 7. In the provided video we show different tasks performed by the gripper. Here, we select representative snapshots from most tasks.

need to counteract the forces exerted by the rubber bands that ensure contact between the cams and the movable part. We estimated a necessary torque of $\Gamma=1,47 \cdot 10^{-3} \mathrm{Nm}$ assuming zero friction between the cam surface and the movable part. We have chosen a Micro Metal HP motor because it is small and can comply with these specifications.

One of bottom fingers is fixed to the wrist to provide the gripper with robustness. The other one has a vertical axis of rotation allowing the finger to abduct/adduct. To leave the bottom plane free to touch the table, this axis of rotation cannot be actuated in a direct way. Therefore, we use a timing belt as a transmission system in order to put the actuator inside the wrist.

We estimated motor maximum torques in worst-case scenarios. Details can be found int [28]. To overcome the high variability in fabric frictions and ensure its control, we accounted for more than twice the computed required torques. In addition, to prevent degrading or burning the motors when the demanded position can't be reached, we chose the torque controlled motors Dynamixel XM430-W210 from the newest families of Dynamixel (X series). However, for the presented experiments, the motors were position controlled. The total weight of the prototype with the handle is $1.112 \mathrm{~kg}$.

\section{EVAluATION OF THE PROTOTYPE}

To evaluate and test the gripper, we have designed 3 sets of tests that for now, they are executed with a person holding 
TABLE II

TIME THE GRIPPER CAN HOLD THE CLOTH WITH EACH EXTRA WEIGHT

\begin{tabular}{|c|c|c|c|c|c|c|c|c|c|c|c|}
\hline Fabric & $\mathrm{Wgt}$ & Fric. Config. & $+7.5 \mathrm{~g}$ & $+22.5 \mathrm{~g}$ & $+48 \mathrm{~g}$ & $+70.5 \mathrm{~g}$ & $+125 \mathrm{~g}$ & $+147.5 \mathrm{~g}$ & $+185 \mathrm{~g}$ & $+202 \mathrm{~g}$ & $+250 \mathrm{~g}$ \\
\hline \multirow{2}{*}{ T-Shirt } & \multirow{2}{*}{$40 \mathrm{~g}$} & Without fric. & $\checkmark$ & $\checkmark$ & $4.3 \mathrm{~s}$ & $x$ & $x$ & $x$ & $x$ & $x$ & $x$ \\
\hline & & With friction & $\checkmark$ & $\checkmark$ & $\checkmark$ & $\checkmark$ & $\checkmark$ & $\checkmark$ & $19.33 \mathrm{~s}$ & $18 \mathrm{~s}$ & $x$ \\
\hline \multirow{2}{*}{ Towel } & \multirow{2}{*}{$60 \mathrm{~g}$} & Without fric. & $\sqrt{ }$ & $\checkmark$ & $\sqrt{ }$ & $\checkmark$ & $\checkmark$ & $1.33 \mathrm{~s}$ & $x$ & $x$ & $x$ \\
\hline & & With friction & $\sqrt{ }$ & $\bar{\checkmark}$ & $\sqrt{ }$ & $\sqrt{ }$ & $\bar{\checkmark}$ & $\checkmark$ & $10.66 \mathrm{~s}$ & $1.33 \mathrm{~s}$ & $x$ \\
\hline \multirow{2}{*}{ Silk } & \multirow{2}{*}{$15 \mathrm{~g}$} & Without fric. & $20 \mathrm{~s}$ & $x$ & $x$ & $x$ & $x$ & $x$ & $x$ & $x$ & $x$ \\
\hline & & With friction & $\checkmark$ & $\checkmark$ & $\checkmark$ & $\checkmark$ & $13.33 \mathrm{~s}$ & $x$ & $x$ & $x$ & $x$ \\
\hline
\end{tabular}

Notes: $\checkmark$ means the grip held for more than 20 seconds, and $X$ means the cloth was slipping out immediately after grasped, while lifting the gripper. Otherwise, we write the average time the cloth took to slip out of the gripper over 3 trials.

the gripper. We leave the evaluation on a robot arm for future work. All the protocols, setup descriptions and videos of the executions can be found in the website ${ }^{2}$ In addition, we show other possible manipulations in an accompanying video, from which we extracted representative snapshots shown in Fig. 7. Quantitative and qualitative evaluation of extra tasks, like fold in the air, is left for future work but an example manipulation is shown in the video.

\section{A. Test 1: Grip strength}

We first tested the grip strength with increasing weights for three different fabrics. A plastic bag was clamped to the cloth to be able to add normalized weights increasingly. With every change of weight, the cloth was grasped three times and time was counted until the cloth slipped out of the gripper, or a maximum of 20 seconds elapsed. If the cloth did not fall off, we marked as a success. If it slipped out immediately, as a fail. The results are shown in Table II. Times shown are the average over 3 trials. Details and pictures of the setup and protocol can be found in the website. As expected, results are very different depending on fabric material, being the $\mathrm{T}$ shirt fabric the one that can hold more weight. This results are not only influenced by the type of material, but also the different thickness they have. In addition, it is interesting that for the towel, there is very little difference between friction and no-friction configuration. The current design has hard silicone material, but these results could be easily improved by using a soft silicone with higher friction coefficient.

\section{B. Test 2: Edge tracing test}

For the second test we performed an edge tracing task without the friction, like in Fig. 7.d), and then we executed a shaking motion either with the friction activated or without it. With this experiment, we want to show, first, that the cloth can slide inside the finger without making contact with the high friction material. Second, that having the ability of activating the friction gives you a better successful rate when performing the shaking motion after reaching the corner. We executed the task for a big tablecloth and a towel from the benchmark in [29], the kitchen towel in Fig. 7-d) and a hemless T-shirt fabric.

\footnotetext{
${ }^{2} \mathrm{http}: / /$ www.iri.upc.edu/groups/perception/\#clothGripper
}

TABLE III

SUCCESS RATE FOR THE SHAKING AFTER TRACING THE EDGE

\begin{tabular}{|c|c|c|c|}
\hline Fabric & Fric. Config. & Num Trials & Success rate \\
\hline \hline \multirow{2}{*}{ Towel } & With friction & 15 & $100 \%$ \\
\cline { 2 - 4 } & Without fric. & 14 & $78.5 \%$ \\
\hline \hline \multirow{2}{*}{ Kitchen cloth } & With friction & 15 & $93.3 \%$ \\
\cline { 2 - 4 } & Without fric. & 10 & $70 \%$ \\
\hline \hline \multirow{2}{*}{ Tablecloth } & With friction & 13 & $84.6 \%$ \\
\cline { 2 - 4 } & Without fric. & 10 & $40 \%$ \\
\hline \hline \multirow{2}{*}{ Hem-less T-shirt fabric } & With friction & 15 & $93.3 \%$ \\
\cline { 2 - 4 } & Without fric. & 13 & $30.7 \%$ \\
\hline
\end{tabular}

In the experiment, the sliding to trace the edge worked well for all the trials, as shown in the videos that can be found in the website. The only difference was in the location of the cloth inside the gripper by the end of the tracing. The results in Table III show the success rate of the shaking motion after tracing the edge, with the fiction activated and without. The cloth was shacked two times, and it was considered successful if the cloth did not slip out of the gripper by the end of the shaking. We could observe that for the towel and the kitchen cloth, even without friction success rate is relatively good. That is because, even without the friction, if the cloth is well inside the gripper the grasp holds. In some cases the hem prevented the cloth to slip. However, if by the end of the edge tracing the cloth is located at a middle point inside the finger, only activating the friction we can secure the grasp. With the kitchen cloth and the hem-less fabric, in one of the executions the cloth ended so far at the fingertip that even with friction, we lost the grip. The tablecloth was big, presenting bigger forces and higher difficulty, but the presence of friction really increased the success rate during shaking. For the hem-less T-shirt fabric, the absence of hems made the cloth slip out very easily unless the friction was activated. In all cases, having the high friction significantly increases the success ratios, meaning that the ability to change friction allows the cloth to slip inside the grip, and also to securely grasp it when needed.

\section{Test 3: Abduction convenience}

Finally, the last task consisted in picking and piling a set of four folded T-shirts from one location to another, on the 


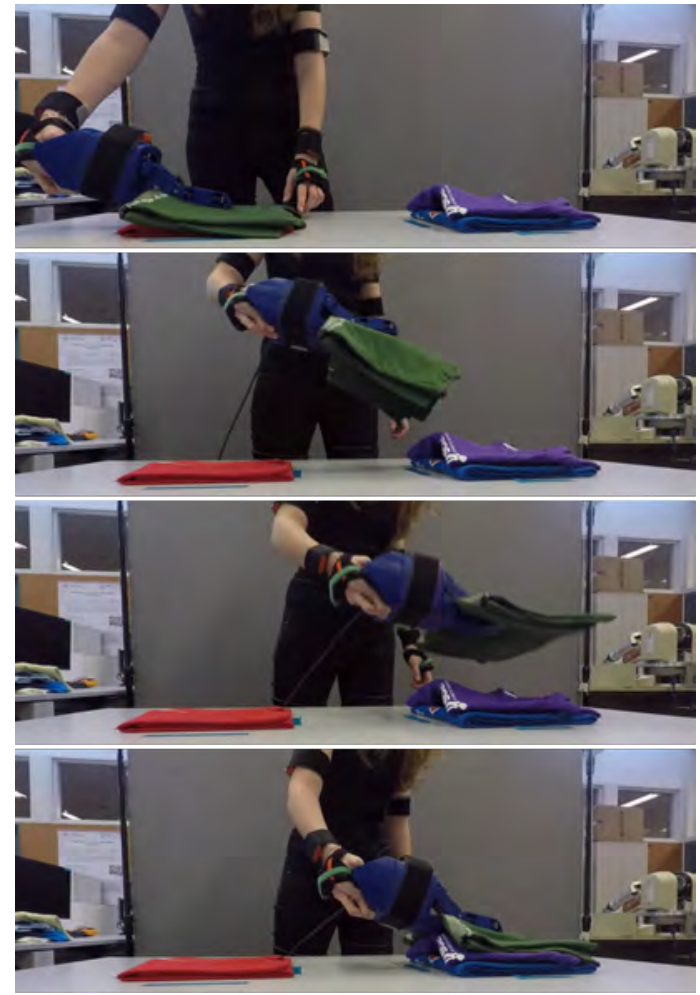

Fig. 8. Frames from pick and pile folded T-shirts without abducted fingers. We performed three trials with abducted fingers and three without, leading to 12 pick and pile motions for each case.

same table as shown in Fig. 8 . We recorded motion data of the person wearing the gripper, and we placed an additional sensor (the prop sensor) attached to the gripper to get position, velocity and acceleration directly from the gripper. In this case, we compare between piling the T-shirt with the bottom fingers closed, that is, with a smaller support surface, versus having the fingers abducted, providing a wider support surface. When placing the T-shirts, humans can achieve good results in terms of not unfolding and leaving the cloth flat in both cases, but the dynamics involved in archiving the same objective are different if the gripper provides more support.

The results of the test are shown in Fig. 9 We normalized the time to align all the 24 trials at the velocity peak that occurs in the placing phase, marked as frame 100 in the plots. We then computed the average curve and standard deviation areas both from the velocity and acceleration profiles. Results show how the velocity and accelerations at the landing phase are significantly different (TTest of 0.0013). In Fig. 10 we show bar plots of the maximum accelerations for each trial. These results clearly show that a higher acceleration is needed when using less support surface, indicating the dynamics of the tasks can be simplified if the gripper provides more support. At the right hand side of Fig. 10, we show maximum accelerations separated by the first to the fourth T-shirt in the pile. We can see that for the first T-shirt the difference is less significant. That is because the surface of the table has very low friction with the cloth and therefore, it is easier to place the T-shirt correctly. Instead,
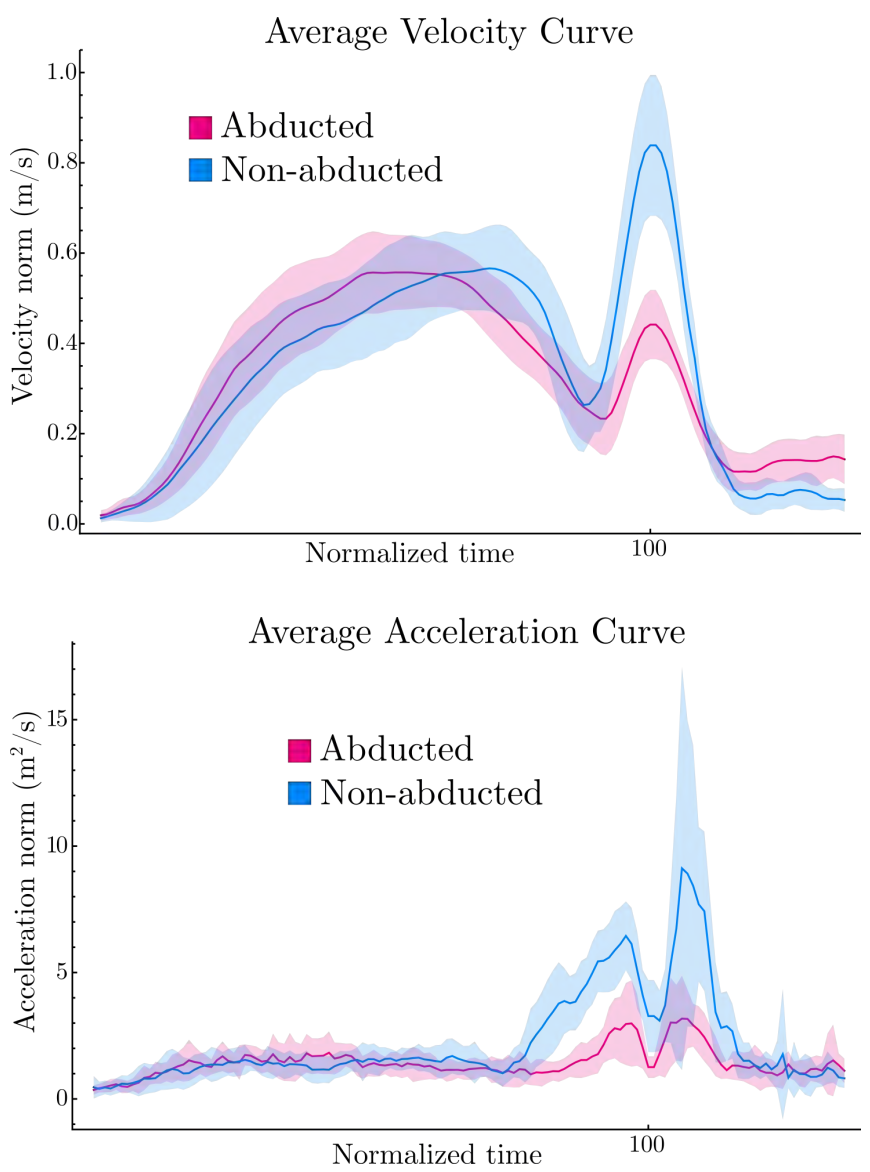

Fig. 9. The mean velocity and acceleration profiles, starting after grasping until landing, with fingers abducted (magenta) or closed (blue).

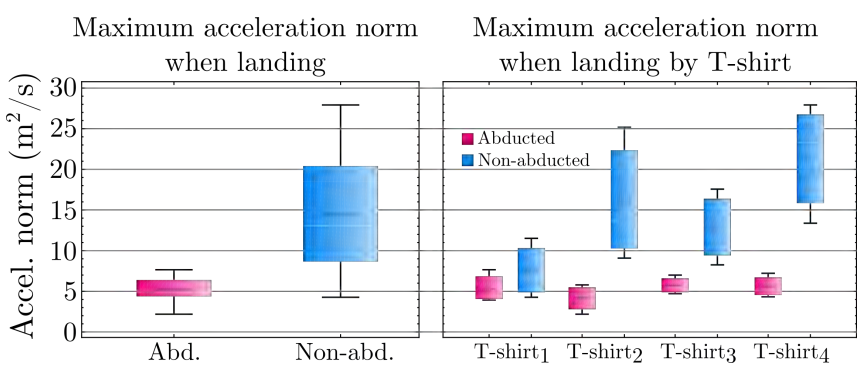

Fig. 10. Maximum acceleration at each trial. On the right, we separate by T-shirt. Magenta corresponds to trials with the fingers abducted, and blue for non-abducted.

placing it on top of another T-shirt requires more dynamic ability.

In conclusion, Table II shows a substantial different behaviour with and without the friction activated. The second test shows how the cloth can easily slip inside the grip without preventing the ability to perform stronger grasps. With high friction alone, edge tracing is not possible. And with only low friction, it is easy to lose grip of the cloth during manipulation. Therefore, having two friction coefficients greatly increases the gripper functionality for cloth manipulation. Finally, the last test shows how increasing the support surfaces when manipulating clothes can simplify the dynamics of a task. 


\section{CONCLUSIONS AND FUTURE WORK}

We have presented the design and development of a gripper prototype designed to execute a variety of cloth manipulation tasks. Motivated by the observation that most of the state-of-the-art cloth manipulation works use only a pinch grasp, we present a design that can deliver a wider variety of grasps to increase functionality and ease some of the tasks, providing more support and requiring simpler monitoring.

Future research includes integrating the gripper in the TIAGo robots that work in our Assisted Living Lab, so as to evaluate the gripper performance when the robots autonomously execute several cloth manipulation tasks (e.g., folding and storing clothes, putting a tablecloth, and making a bed). In order to complete other envisaged tasks in the Lab (e.g., emptying the dishwasher and setting up a table), we need the gripper to be able to manipulate rigid objects as well. Thus, in our gripper design, we plan to add an additional degree of freedom at the top finger to allow for rigid-object grasping, as mentioned in Section 2. Moreover, we are also planning to integrate touch sensors on the gripper, allowing the assess if a grasp has been successful without vision.

Together with the latest advances in cloth state estimation and grasping point detection, progress on hardware design is necessary to increase dexterity and allow successful cloth manipulation executions. In this direction, our gripper shows increased manipulation skills and can ease the execution of several relevant cloth manipulations.

\section{ACKNOWLEDGEMENTS}

Authors thank Dr. Patrick Grosch for his valuable insights on the mechanical design implementation, Sergi Hernández for his work on the electronics of the gripper and Dr. Massimo Cenciarini for his support on the human subjects data analysis.

\section{REFERENCES}

[1] J. Sanchez, J.-A. Corrales, B.-C. Bouzgarrou, and Y. Mezouar, "Robotic manipulation and sensing of deformable objects in domestic and industrial applications: a survey," Int. J. of Rob. Res., vol. 37, no. 7, pp. 688-716, 2018.

[2] A. Verleysen, M. Biondina, and F. wyffels, "Video dataset of human demonstrations of folding clothing for robotic folding." https:// adverley.github.io/folding-demonstrations/

[3] J. Maitin-Shepard, M. Cusumano-Towner, J. Lei, and P. Abbeel, "Cloth grasp point detection based on multiple-view geometric cues with application to robotic towel folding," in IEEE Int. Conf. on Rob. and Auto., pp. 2308-2315, 2010.

[4] S. Miller, J. Van Den Berg, M. Fritz, T. Darrell, K. Goldberg, and P. Abbeel, "A geometric approach to robotic laundry folding," Int. J. of Rob. Res., vol. 31, no. 2, pp. 249-267, 2012.

[5] Y. Li, Y. Yue, D. Xu, E. Grinspun, and P. K. Allen, "Folding deformable objects using predictive simulation and trajectory optimization," in IEEE/RSJ Int. Conf. on Intelligent Robots and Systems, pp. 6000-6006, 2015.

[6] A. Doumanoglou, J. Stria, G. Peleka, I. Mariolis, V. Petrik, A. Kargakos, L. Wagner, V. Hlavac, T.-K. Kim, and S. Malassiotis, "Folding clothes autonomously: A complete pipeline," IEEE Transactions on Robotics, vol. 32, no. 6, pp. 1461-1478, 2016.
[7] H. Yuba, S. Arnold, and K. Yamazaki, "Unfolding of a rectangular cloth from unarranged starting shapes by a dual-armed robot with a mechanism for managing recognition error and uncertainty," Advanced Robotics, vol. 31, no. 10, pp. 544-556, 2017.

[8] J. Borràs, G. Alenya, and C. Torras, "A grasping-centered analysis for cloth manipulation," IEEE Transactions on Robotics (to appear), 2020.

[9] P. Koustoumpardis and N. Aspragathos, "A review of gripping devices for fabric handling," in Int. Conf. on Intelligent Manipulation and Grasping, 2004.

[10] T.-H.-L. Le, M. Jilich, A. Landini, M. Zoppi, D. Zlatanov, and R. Molfino, "On the development of a specialized flexible gripper for garment handling," Journal of Automation and Control Engineering, vol. 1, no. 3, pp. 255-259, 2013.

[11] P. N. Koustoumpardis, K. X. Nastos, and N. A. Aspragathos, "Underactuated 3-finger robotic gripper for grasping fabrics," in Int. Conf. on Robotics in Alpe-Adria-Danube Region, pp. 1-8, 2014.

[12] E. Ono and K. Takase, "On better pushing for picking a piece of fabric from layers," in IEEE Int. Conf. on Robotics and Biomimetics, pp. 589-594, 2007.

[13] F. Von Drigalski, T. Ogasawara, J. Beltran, D. Yoshioka, W. Yamazaki, S.-G. Cho, M. Gall, P. M. U. Eljuri, V. Hoerig, M. Ding, et al., "Naist openhand m2s: A versatile two-finger gripper adapted for pulling and tucking textile," in IEEE Int. Conf. on Robotic Computing (IRC), pp. 117-122, 2017.

[14] K. Salleh, H. Seki, Y. Kamiya, and M. Hikizu, "Inchworm robot grippers for clothes manipulation," Artificial Life and Robotics, vol. 12, no. 1-2, pp. 142-147, 2008.

[15] K. Salleh, H. Seki, Y. Kamiya, and M. Hikizu, "Tracing manipulation of deformable objects using robot grippers with roller fingertips," in Int. Joint Conf. SICE-ICASE, pp. 5882-5887, 2006.

[16] M. Shibata, T. Ota, Y. Endo, and S. Hirai, "Handling of hemmed fabrics by a single-armed robot," in IEEE Int. Conf. on Automation Science and Engineering, pp. 882-887, 2008.

[17] A. Karakerezis, Z. Doulgeri, and V. Petridis, "A gripper for handling flat non-rigid materials," in Automation and Robotics in Construction XI, pp. 593-601, 1994.

[18] A. J. Spiers, B. Calli, and A. M. Dollar, "Variable-friction finger surfaces to enable within-hand manipulation via gripping and sliding," IEEE Rob. and Auto. Let., vol. 3, no. 4, pp. 4116-4123, 2018.

[19] K. P. Becker, N. W. Bartlett, M. J. Malley, P. M. Kjeer, and R. J. Wood, "Tunable friction through constrained inflation of an elastomeric membrane," in IEEE Int. Conf. on Rob. and Auto., pp. 4352-4357, IEEE, 2017.

[20] S. Nojiri, K. Mizushima, Y. Suzuki, T. Tsuji, and T. Watanabe, "Development of contact area variable surface for manipulation requiring sliding," in IEEE Int. Conf. on Soft Robotics, pp. 131-136, 2019.

[21] Q. Lu, A. B. Clark, M. Shen, and N. Rojas, "An origami-inspired variable friction surface for increasing the dexterity of robotic grippers," IEEE Rob. and Auto. Let., 2020.

[22] R. R. Ma, A. Spiers, and A. M. Dollar, "M2 gripper: Extending the dexterity of a simple, underactuated gripper," in Advances in reconfigurable mechanisms and robots $I I$, pp. 795-805, Springer, 2016.

[23] T. Iberall, "Opposition space as a structuring concept for the analysis of skilled hand movements," Generation and modulation of action patterns, vol. 15, pp. 158-173, 1986.

[24] T. Iberall, C. Torras, and C. MacKenzie, "Parameterizing prehension: A mathematical model of opposition space," in COGNITIVA Symp. on At the crossroads of artificial intelligence, cognitive science, and neuroscience, pp. 635-642, 1991.

[25] Y. Moriya, D. Tanaka, K. Yamazaki, and K. Takeshita, "A method of picking up a folded fabric product by a single-armed robot,' ROBOMECH Journal, vol. 5, no. 1, p. 1, 2018.

[26] C. Bersch, B. Pitzer, and S. Kammel, "Bimanual robotic cloth manipulation for laundry folding," in IEEE/RSJ Int. Conf. on Intelligent Robots and Systems, pp. 1413-1419, 2011.

[27] A. Colomé and C. Torras, "Dimensionality reduction for dynamic movement primitives and application to bimanual manipulation of clothes," IEEE Transactions on Robotics, vol. 34, no. 3, pp. 602-615, 2018.

[28] S. Donaire, "Mà robòtica multifuncional per a tasques domèstiques," B.S. thesis, Universitat Politècnica de Catalunya, 2019.

[29] I. Garcia-Camacho, M. Lippi, et al., "Benchmarking bimanual cloth manipulation," IEEE Rob. and Auto. Let., vol. 5, no. 2, pp. 1111-1118, 2020. 